\title{
Search for millicharged particles at the LHC with the milliQan prototype
}

\author{
Matthew Citron;* \\ University of California, Santa Barbara, California 93106, USA \\ E-mail: mcitron@cern.ch
}

We report on the results of a recent search for fractionally charged particles using a data sample of $37.5 \mathrm{fb}^{-1}$ proton-proton collisions at $\sqrt{s}=13 \mathrm{TeV}$ provided by the CERN Large Hadron Collider in 2018. This search was carried out with a prototype scintillator-based detector, which allows the first sensitivity to particles with charges $\leq 0.1 \mathrm{e}$ at a hadron collider. The existence of new particles with masses between 20 and $4700 \mathrm{MeV}$ is excluded at $95 \%$ confidence level for charges between $0.006 \mathrm{e}$ and $0.3 \mathrm{e}$, depending on their mass. New sensitivity is achieved for masses larger than $700 \mathrm{MeV}$. The successful operation of the milliQan prototype provides crucial lessons for the design of the full milliQan detector.

40th International Conference on High Energy physics - ICHEP2020

July 28 - August 6, 2020

Prague, Czech Republic (virtual meeting)

\footnotetext{
${ }^{*}$ Speaker
} 


\section{Motivation}

In recent years the possibility that dark matter is not a single particle, but instead a diverse set of particles with as complex a structure in their sector as the Standard Model, has risen in prominence. In the case that the dark sector contains a massless dark photon, the main physical effect is that new dark sector particles that couple to the dark photon will have a small electric charge, a "millicharge" [1]. The parameter space of millicharged particles (herein denoted $\chi$ ) is robustly constrained by direct searches, however, indirect observations can be evaded by adding extra degrees of freedom, which can readily occur in minimally extended dark sector models. In particular, the parameter space $1<m_{\chi}<100 \mathrm{GeV}$ is largely unexplored by direct searches. Such particles may be produced copiously at the LHC, however, general purpose detectors such as CMS and ATLAS are insensitive to new particles with $Q<0.1 e$. This is because the interaction strength of a particle of charge $\mathrm{Q}$ is reduced by factor $(Q / e)^{2}$ compared to a particle of the same mass with charge $e$. A dedicated experiment, the milliQan detector [2], is therefore required to provide sensitivity to millicharged particles at the LHC.

\section{The milliQan prototype}

The proposed milliQan detector is designed to achieve sensitivity to low charge particles by making use of a array of long scintillator bars $(5 \mathrm{~cm} \times 5 \mathrm{~cm} \times 80 \mathrm{~cm})$ aligned with the CMS interaction point (IP), where the LHC beams are brought together for collisions. A large sensitive volume of scintillator is required to allow sensitivity to the small energy deposition of a low charge particle. To provide sensitivity to charges as low as $0.001 e$, each scintillator bar must be coupled to a Photomultiplier Tube (PMT) that can detect a single scintillation photon. As shown in Fig. 1a, the proposed detector is comprised of multiple layers of scintillator bars such that a millicharged particle produced at the CMS IP will travel upwards through the detector, depositing energy in each layer. Backgrounds from a variety of sources can be suppressed by requiring a signal to be observed in each layer that arrives within a small time window and that points towards the CMS IP. The detector is proposed to be located in the CMS experimental site at a distance of $33 \mathrm{~m}$ from the CMS IP, including $17 \mathrm{~m}$ of rock that shields the detector from particles produced in LHC collisions. In the CMS coordinate system the milliQan detector site is located at an azimuthal angle of $43^{\circ}$ and a pseudorapidity of 0.1 .

In order to characterise the backgrounds and prove the feasibility of a dedicated scintillatorbased detector, a small prototype experiment was installed. The milliQan prototype consists of eighteen scintillator bars arranged in three layers. In addition, lead bricks and scintillator "slabs" were placed between each layer and at the front and back of the milliQan prototype to shield radiation and identify through-going particles, and thin scintillator "panels" were placed around each layer to tag cosmic muons and shower particles. A diagram showing the milliQan prototype can be seen in Fig. 2a, while Fig 2b shows a photograph of the milliQan prototype during installation.

The milliQan detector ran successfully from March 2018 to May 2019, providing crucial experience in the operation of a dedicated scintillator-based detector. The $37.5 \mathrm{fb}^{-1}$ of data collected by the milliQan prototype was used to fully calibrate the detector and carry out a search for fractionally charged particles with world-leading sensitivity [3]. 


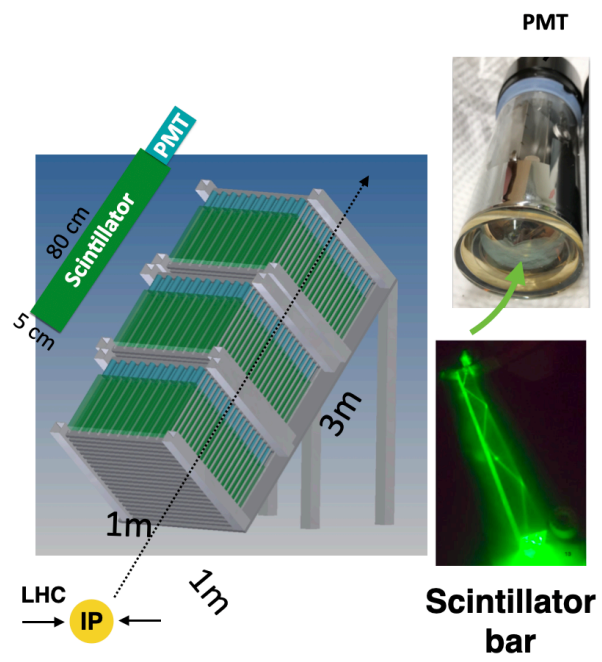

(a)

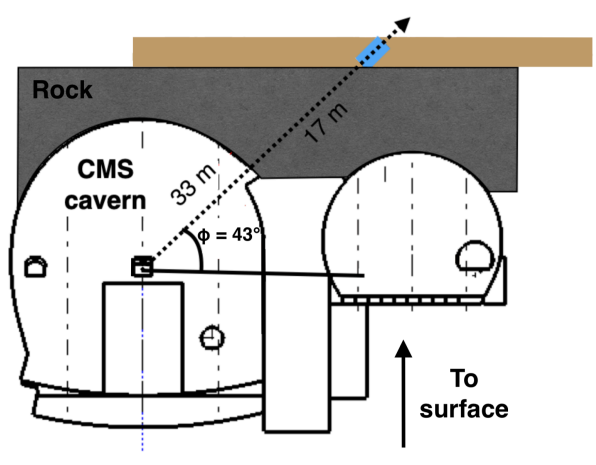

(b)

Figure 1: (a) A diagram of the milliQan detector, a representative scintillator bar, and a PMT (b) Diagram showing the detector location

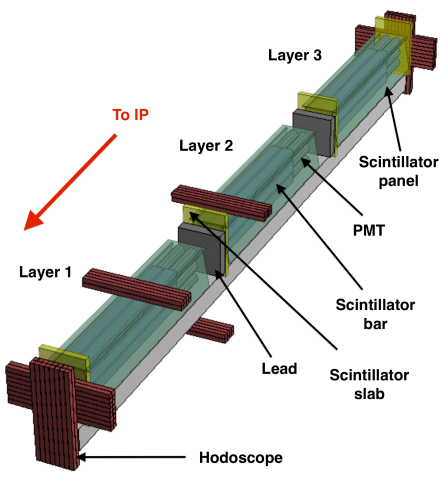

(a)

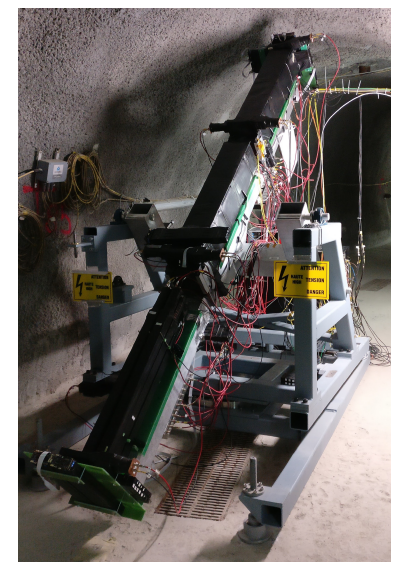

(b)

Figure 2: (a) Diagram of the milliQan prototype components (b) Photograph of the milliQan prototype during installation

\section{Simulation}

In order to evaluate the sensitivity of the detector we generate the production of millicharged particles from a wide array of sources. Where available, the differential cross section of the parent particles is taken from the latest measurements from CMS, ATLAS and LHCb. The branching fraction to millicharged particles is then evaluated. The resulting production cross sections from the processes considered is summarised in Fig 4a.

In addition to signal processes, we also generate muon production in proton-proton collisions and through cosmic rays. Particles generated at the CMS IP are propagated through a simplified model of the CMS detector and the rock up to $2 \mathrm{~m}$ before the milliQan prototype face. This is 
achieved using a fourth-order Runge-Kutta integration that takes into account the magnetic field, energy loss and multiple scattering. At this point the momentum four-vector is passed to a full Geant4 [4] simulation of the cavern and milliQan detector material. The response per channel is calibrated using measurements in data as detailed in Section 4. Figure 3a shows a comparison of the PMT response in data and simulation for shower particles from muons, which are shown to agree well. The overall rate of muons originating from the beam is observed to be $0.20 \pm 0.01$ muons $/ \mathrm{pb}^{-1}$, in agreement with the prediction of $0.25 \pm 0.08$ muons $/ \mathrm{pb}^{-1}$.

\section{Calibration}

The pulse size must be calibrated to determine the PMT response for a particle of a particular charge. This calibration is carried out by first measuring the average size of a single photoelectron (SPE) in each PMT. This is done by isolating pulses from late-arriving photons and locating the peak of the pulse area distribution. The number of photoelectrons $\left(n_{\mathrm{pe}}\right)$ for cosmic muons is then determined for each PMT by taking the ratio of the average pulse area for a cosmic muon over the SPE area. This value is then used to correct the simulation of the response of each scintillator bar, slab and panel. The measured response to a millicharged particle traversing a scintillator bar corresponds to a mean value of $n_{\mathrm{pe}}=1$ for charges of $Q / e \sim 0.004-0.007$, depending on the PMT.

The PMT timing calibration is carried out using a combination of beam and cosmic muons such that a through-going particle from the CMS IP should have the same time value for each bar, slab and panel. The calibrated time difference between hits in the outer layers of the detector is shown in Fig. 3b. The resolution in the time difference between layers is calculated to be $4 \mathrm{~ns}$.

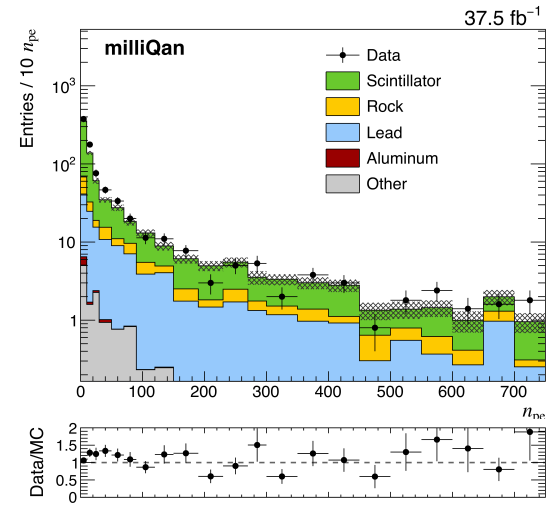

(a)

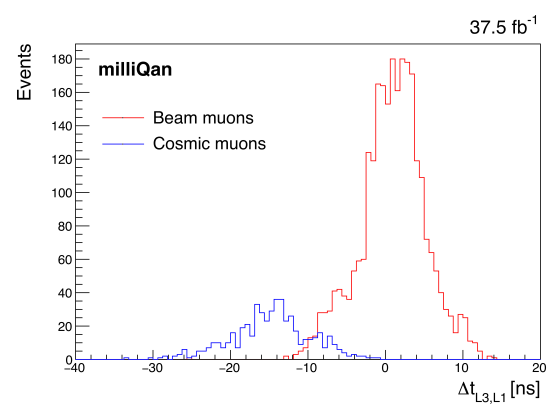

(b)

Figure 3: (a) Comparison of the $n_{\text {pe }}$ distribution for muon shower particles (b) Calibrated time difference between bars in the first and third layer of the milliQan prototype for beam and cosmic muons

\section{Search for millicharged particles}

A millicharged particle will produce a hit in each of the three layers of the milliQan prototype. There are a number of sources of background that can produce such a signature, including overlap of thermal dark rate pulses, shower particles created by cosmic muons, and radiation in the cavern. 
A wide range of selection are applied to reject such background processes. These include a requirement of exactly one hit in each layer that form a pointing path to the CMS IP and arrive within a $15 \mathrm{~ns}$ time window. Together with requirements on the pulse quality, these selection reduce the background passing selection by over five orders of magnitude. Events are finally categorised into five signal regions depending on the $n_{\text {pe }}$ in the bars and the number of slabs with a pulse. This provides wide sensitivity to a range of masses and charges [3].

Residual backgrounds are predicted using the pass/fail ratio of the timing requirement in events with a hit in each layer, satisfying signal requirements except that the bars do not form a pointing path towards the CMS IP, and then multiplying it by the number of events failing the timing selection that form a pointing path towards the CMS IP. This method relies on dominant backgrounds being independent of the pointing path requirement. As the backgrounds do not originate from beam processes, this independence is confirmed in data collected during periods the LHC beams are not producing collisions. The level of agreement observed is used to define a systematic uncertainty in the prediction.

The search is interpreted using the signal simulation described in Section 3. A range of systematic uncertainties are included to account for any mismodelling of the detector response, the limited statistical sample, and uncertainties in the signal production cross section and propagation. The resultant observed upper limits at 95\% confidence level are shown in Fig. 4b. New sensitivity is achieved for $\chi$ masses above $700 \mathrm{MeV}$.

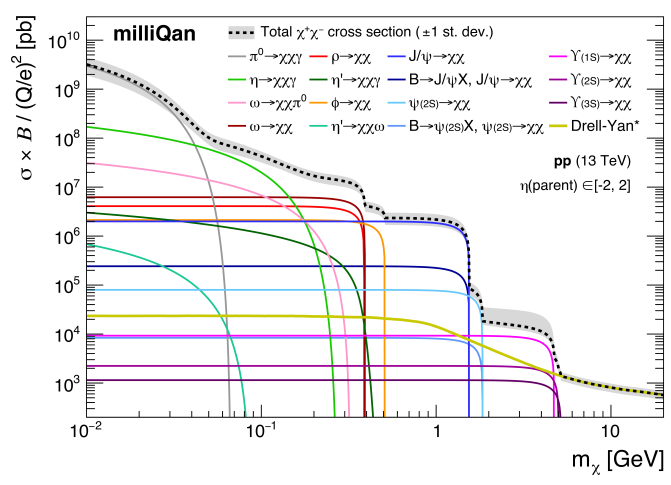

(a)

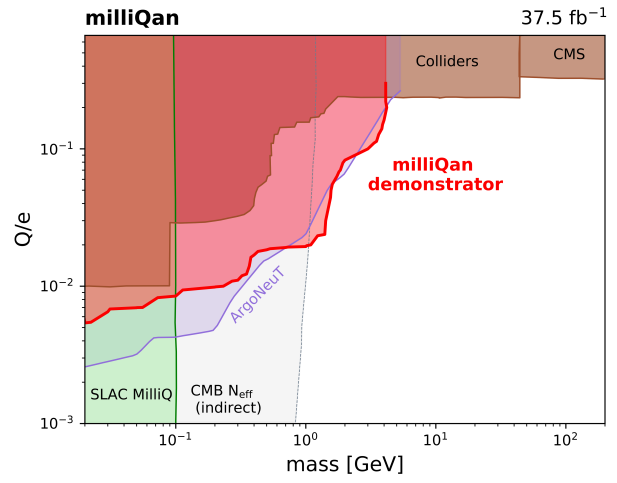

(b)

Figure 4: (a) Production cross section times branching ratio for millicharged particles (b) Exclusion limits in the mass and charge plane for the milliQan prototype compared to previous constraints

\section{Future plans}

The original detector design proposed in Ref. [2], which has three layers of scintillator bars, was motivated by the assumption that dark rate pulses in the PMT provide the dominant background. The data collected by the milliQan prototype has shown that correlated backgrounds from processes such as showers from cosmic muon interactions in the cavern are equally important. This motivates an update to a four layer design that allows such backgrounds to be mitigated. The background rejection has been confirmed through dedicated runs of the milliQan prototype in a four layer 
configuration. The design of the full milliQan detector is shown in Fig. 5 and comprises 864 bars arranged in modules of $2 \times 2 \times 4$ bars. The use of improved PMTs as well as the enhanced active veto capabilities of the larger detector will allow significantly improved sensitivity for millicharged particles at the HL-LHC. In preparation, the milliQan collaboration has received funding to build an intermediate four layer detector for the upcoming Run 3 of the LHC.
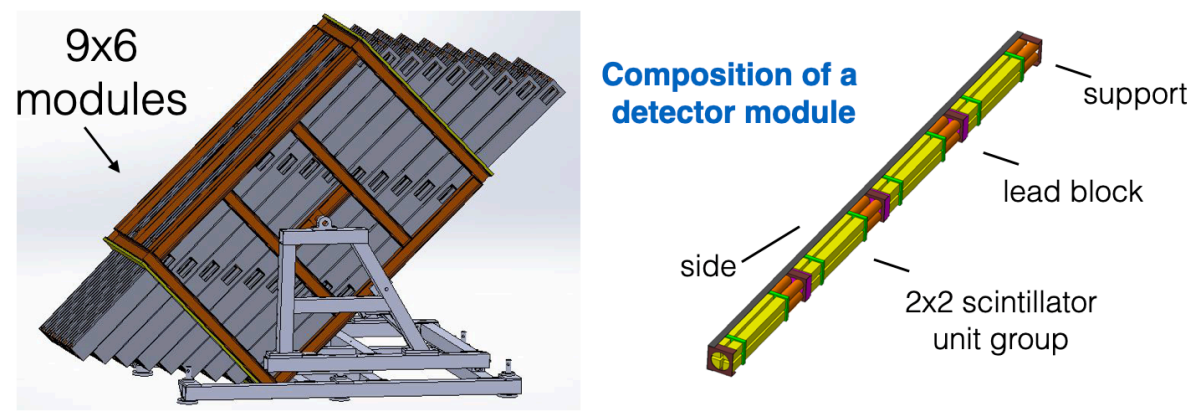

Figure 5: The updated design of the full milliQan detector for the HL-LHC

\section{Conclusions}

The milliQan prototype has been deployed to allow the first search for millicharged particles at a collider. A data sample of $37.5 \mathrm{fb}^{-1}$ proton-proton collisions at $\sqrt{s}=13 \mathrm{TeV}$ has been analysed and no excess over the background prediction observed. The existence of new particles with masses between 20 and $4700 \mathrm{MeV}$ is excluded at $95 \%$ confidence level for charges varying between $0.006 e$ and $0.3 e$, depending on their mass. New sensitivity is achieved for masses larger than $700 \mathrm{MeV}$. The feasibility of a scintillation-based detector at the LHC has been confirmed and important lessons provided for future detectors.

\section{Acknowledgements}

This work was supported by the US Department of Energy under award number DESC0011702.

\section{References}

[1] B. Holdom, “Two U(1)'s and Epsilon Charge Shifts.” Phys. Lett. B 166 (1986) 196 [doi:10.1016/0370-2693(86)91377-8].

[2] A. Ball, J. Brooke, C. Campagnari, et al., "A Letter of Intent to Install a Milli-Charged Particle Detector at LHC P5.” 2016. [arXiv:1607.04669].

[3] A. Ball, G. Beauregard, J. Brooke, et al., "Search for millicharged particles in proton-proton collisions at $\sqrt{s}=13 \mathrm{TeV}$." Physical Review D 102 (2020) [arXiv:2005.06518].

[4] S. Agostinelli, J. Allison, K. Amako, et al., "Geant4-a simulation toolkit." Nucl. Instrum. Methods A 506 (2003) 250 [doi:10.1016/S0168-9002(03)01368-8]. 